\title{
Infant Botulism
}

National Cancer Institute

\section{Source}

National Cancer Institute. Infant Botulism. NCI Thesaurus. Code C128343.

Botulism that is caused by contact with spores of Clostridial bacteria, which subsequently grow in the intestine and release toxin. 